\title{
Spatial Distribution Analysis with Capture Effect of a Mobile S-ALOHA Network
}

\author{
David Covarrubias ${ }^{1}$, Silvia Ruiz, Joan Huguet and Joan Olmos \\ Signal Theory and Communications Department \\ Polytechnic University of Catalonia (UPC) \\ Campus Nord-D4, Jordi Girona Salgado 1-3, 08034 Barcelona, Spain \\ E-mail: dacoro, silvia, joan, olmos @xaloc.upc.es, fax (+34-93)401-7200
}

\begin{abstract}
The throughput performance of a mobile S-ALOHA network can be improved considering the capture phenomenon, which also depends on the spatial distribution of the mobiles within the cell. We have studied the capture probabilities that arise in a mobile radio scenario in the presence of fading and shadowing, and considering both, uniform and non-uniform spatial distribution models. In particular we were interested in the limit behaviour of these models, which has been shown to be directly related to the capture probability. This analysis allows a quantitative comparison of three spatial distribution models for mobile users under real mobile channels. The use of an exponential backoff retransmission algorithm is considered. With these assumptions the performance of the anarchic ALOHA is improved considerably obtaining higher throughput values with stabilised behaviour and lower delays values.
\end{abstract}

\section{INTRODUCTION}

Slotted ALOHA (S-ALOHA) is a random multiple access protocol well known in mobile networks. Mobile terminals transmit packets over a common radio channels in predefined time slots, but without any mutual control or centralised regulation except for a positive acknowledgements of reception. If two or more terminals transmit simultaneously, a packet collision occurs, and this mutual interference may result in a loss of packets. So to minimise these losses and achieve higher throughput values and stabilised behaviour we have taken into account several aspects in a mobile real channel conditions:

Retransmission Algorithm: it is one of the important issues in S-ALOHA. The original S-ALOHA system uses a constant value of $p$ (retransmission probability) and is inherently unstable under high traffic conditions [1],[2].

Channel Effects: Propagation in the mobile channel is described by means of three effects, attenuation due to distance (to calculate mean received power level), shadowing (to

'also with CICESE Mexican research centre and recipient of a CONACYT Ph.D. grant calculate slow variations over the mean received power) and Rayleigh fading due to multipath (to calculate fast variations of instantaneous received power) [3], [4].

Capture effect. Most receivers have the ability to capture the strongest of two or more signals, provided that the ratio of the signal power over the total interference power exceeds some value, which is called the capture ratio [5].

Spatial Distribution. Since the mobile terminals will usually be at different distances from Base Station (BS) their received signal powers will be different. Thus the Capture Probability depends on the spatial distribution of the mobiles around the BS [6],[7]. It is assumed that the locations of the mobiles relative to the BS are determined by the distance and are a random variable. Considering the BS centred in a circle of unit radius we study the capture probabilities that arise in a mobile radio scenario,

The main features of this paper are: first, as the effect of the distribution of the interferes is of primary concern, the role of spatial distributions is clearly identified by considering three different models. Secondly, spatial distribution effects over capture probability have been explicitly evaluated for the three models. Finally, the influence of receiver capture on the performance of S-ALOHA in the presence of noisy and fading channel has been calculated.

The paper is organised as follow. In section II the mobile system model is presented taking into account noise, fading and shadowing effects. In section III the analysis of the spatial distribution models is presented. In section IV numerical results are given using a computer simulation in a real communications scenario. The discussion and conclusions are summarised in section $\mathrm{V}$.

\section{MOBILE SYSTEM MODEL}

We consider a mobile S-ALOHA network with finite number of users (due to the exponential backoff scheme) generating Poisson distributed data traffic being only the uplink considered in the study. The mobile channel is characterised by 
noise, lognormal shadowing and Rayleigh fading.

\section{A. Channel Effects [3],[4]}

Mean received power: We assume that the mean received power follows an inverse distance dependence law being $\alpha$ the rate decreasing. Along the simulation we use $\alpha=4$ (plane earth model). Then the received power follows the equation:

$$
P_{R}=K \cdot r^{-\alpha}
$$

being $\mathrm{r}$ the distance between transmitter and receiver and $\mathrm{K}$ a parameter which depends on transmitted power, frequency, antenna gains and elevation. Along the simulation we consider that the system is limited by interference as is usual in mobile communications.

Shadowing: The lack of visibility between BS and mobile terminal makes the signal fade out. The variation of received power (local mean power $\mathrm{P}_{\mathrm{m}}$ ) due to slow fading is lognormally distributed. Gaussian pdf is characterised by mean value and standard deviation. Mean value is obtained through the plane earth model and typical values are used for the standard deviation.

Rayleigh fading: Due to multipath propagation the signal is affected by Rayleigh fading, which causes the instantaneous envelope of the received signal to be Rayleigh distributed. Thus the instantaneous received power $\mathrm{P}$ is exponentially distributed about the local mean power $P_{m}$. The exponential pdf is:

$$
p_{s}(P)=\frac{1}{P_{m}} \cdot e^{-\frac{P}{P_{m}}}
$$

In the simulation we consider only Slow Rayleigh Fading, this is a constant signal level over the duration of a packet is considered (packet duration is much shorter than the coherence time of the mobile channel).

\section{B. Capture Effect [5-7]}

Is the phenomenon whereby a remote user's transmission packet is received successfully in the presence of some other contending packets. Due to differences in received power levels the transmitted packet of the stronger user can be successfully received although other packets from other users were also transmitted.

The probability that the desired packet captures the receiver is called Capture Probability $\left(\mathrm{P}_{\text {capt }}\right)$. In the presence of $I(I \geq 1)$ interfering packets, this probability is obtained by comparing the power of the desired packet with total interference power:

$$
P_{\text {capt }}=\operatorname{Pr}\left(P_{d}\left(\sum_{j=1}^{I} P_{\mathrm{int} j} j\right)^{-1}>R\right)
$$

being $P_{d}$ and $P_{i n t}$ the powers of the desired and interference packets respectively and $R$ the Capture Ratio. The range of $R$ is from 1 (Perfect Capture) and $\infty$ (No capture).

\section{Retransmission Algorithm}

An ideal S-ALOHA is unstable under high traffic conditions. There are different methods to stabilise the S-ALOHA system by controlling $p$ dynamically. Stability has been proved for several control schemes in which the retransmission probabilities are a function of the backlog (mobile terminals with packets to retransmit are said to be in backlog state). In this work we choose the method known as Exponential Backoff Scheme (EB) suggested by [8] which directly estimates $p$ according to the transmission result of the previous slot. EB scheme is comparable to ideal S-ALOHA for low traffic but offers higher stabilised throughput values in high traffic conditions [9].

\section{SPATIAL DISTRIBUTION MODELS}

As we mentioned before the throughput performance of a mobile S-ALOHA network is improved under real channel propagation conditions. As the capture phenomenon also depends on the spatial distribution of the mobiles within the cell, a proper study of this parameter is of importance.

We have applied uniform and no-uniform spatial distributions. In the last case we have considered Bell-Shaped and Unimodal Distributions. The integral transforms of the probability density function (pdf) of the spatial distributions have been obtained for each model. This is a necessary tool for analysing the channel capacity (capture probability) in the presence of a population of distributed mobile users.

\section{A. Uniform}

It is the most simple, most used and most tractable model. In a uniform distribution the spatial traffic density is uniformly distributed around the BS and then the number of mobiles per unit area is constant (does not depend on the distance to BS) [10]. Perhaps the limitation of this model is that considering the same traffic per unit area at close or far distance of the BS is sometimes not realistic. Most of the traffic comes usually from users near the BS because users in the limits of coverage could be served by another BS.

Considering the BS centred in a circle of unit radius, normalising the traffic on the channel to unity and considering the use of an omnidirectional antenna at the BS the probability density function (pdf) of the users distribution at a distance $y$ is [11]:

$$
f_{u}(y)=2 y \quad 0 \leq y \leq 1
$$

as can be seen the number of users increases as the distance to BS grows, giving then a constant users distribution per unit 
area. Also it can be seen that the mobiles can not be arbitrarily close to the BS.

\section{B. Bell Shape}

In this case the spatial traffic density models a virtually constant traffic density inside the unit circle with a quickly decay outside. The name of this distribution is given by the shape of the pdf of the users distribution at a distance $y$. In this case the density of mobiles per unit area is not constant and a higher number of mobiles is considered near the BS than at the limits of the coverage area. This could stand for the external mobiles being served by another BS. The pdf of the distance $y$ is [12].

$$
f_{b}(y)=2 y \cdot e^{-\pi \cdot \frac{y^{4}}{4}} \quad y>0
$$

\section{C: Unimodal}

This is also a non uniform spatial traffic density where the mobiles are distributed within a narrow ring-shaped region surrounding the BS. The shape of the probability density of an interfere shows a single peak at $\mathrm{y}=\mathrm{y}_{0}$ where $\mathrm{y}$ is the distance from any user to BS, and the radius $y_{0}$ is the location of interferes in the neighbourhood of the ring. The pdf of the distance $y$ is [13]:

$$
f_{u n}(y)=\frac{5 y_{0}^{4}}{y^{5}} \cdot e\left[-\frac{5}{4} \cdot\left(\frac{y_{0}}{y}\right)^{4}\right] \quad y>0
$$

It must be understood that the unimodal distribution is considered only to study the throughput and delay parameters in the worst case, because this distribution does not represent a real environment. In other words, we would know if there is any chance for a mobile to be captured due only to shadowing and Rayleigh fading.

\section{NUMERICAL RESULTS}

To analyse a real environment the Capture Probability given by the following expression should be solved:

$$
\begin{aligned}
P_{\text {capt }} & =B^{2} \int_{0}^{\infty \infty} \int_{0}^{\infty} \int_{0} p d f(y) \cdot \frac{1}{\left(R y^{4} v+u\right) v} \cdot e^{\frac{-(A-\log v)^{2}}{2 \sigma^{2}}} . \\
& e^{-\frac{(A-\log u)^{2}}{2 \sigma^{2}} d y d u d v}
\end{aligned}
$$

where pdf(y) represents the spatial distribution, $b$ is the upper limit depending on the specific distribution, and $\sigma$ is the standard deviation

$$
A=-\log P_{R}+\frac{\sigma^{2}}{2 \log e} \quad B=\frac{\log e}{\sigma \cdot \sqrt{2 \pi}}
$$

The throughput ( $S$ ) follows the equation $S=G \cdot P$, being $G$ the channel traffic and $\mathrm{P}$ the probability that a packet is successfully transmitted

$$
P=e^{G \cdot\left(P_{\text {capt }}-1\right)}
$$

It is difficult to solve these integrals by both analytical and numerical methods, and in any case only the throughput would be obtained, so we have used computer simulation.

The parameters of the simulation are the following:

- We consider 50 mobile terminals

- At each terminal packets are generated according to a Bernoulli process with generation probabilities from $10^{-3}$ to 1. For each generation probability 10,000 slots have been transmitted in order to obtain mean values.

- A terminal cannot generate a new packet until the actual packet has been transmitted.

- A minimum value for the retransmission probability $p$ is considered $\left(p_{\min }=q^{9}\right)$. Along all the simulations $q=0.5$.

- Channel parameters are: Log-normal statistics with $\sigma=5$ $\mathrm{dB}$, and plane earth propagation model $(\alpha=4)$.

- Location of interferes at $y_{0}=0.5$

Computer simulations have been made, obtaining throughput curves shown in Figs. 1-3. The channel throughput increases when the capture ratio decreases from $R=10$ (nearly no-capture) to $\mathrm{R}=2$ (typical value of almost perfect capture). As a reference, we also include the throughput when no channel is considered which is named S-ALOHA ideal or theorist. In this case the retransmission probability is assumed to be equal to new packet generation probability (as is usually assumed in many studies).

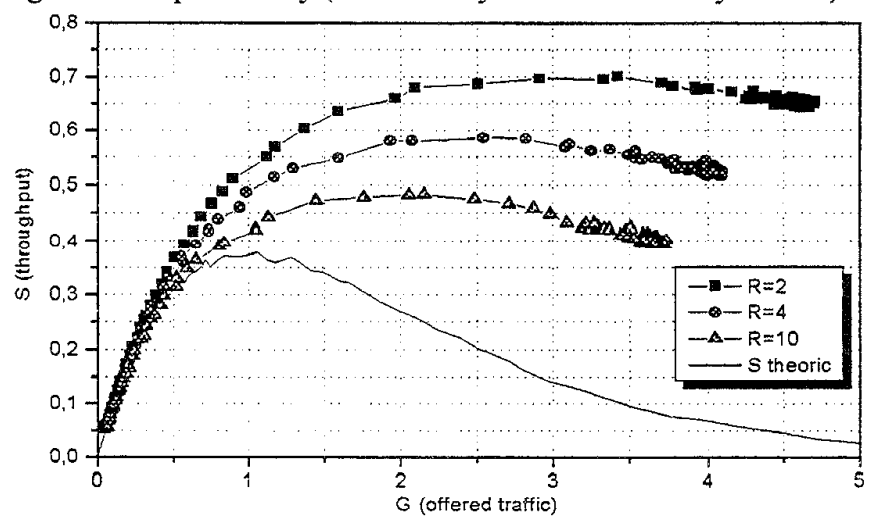

Figure 1. Throughput versus Offered Load for a Uniform Spatial Distribution for different capture ratio. 
As was expected, Fig.2, the Bell-Shaped spatial distribution offers the highest throughput under high-traffic conditions with a very stable behavior when compared with the other distributions. This was expected because this distribution reduces the probability of a mobile terminal being "too far" from the BS (in the coverage limit). As we know, mobiles in the limit of the coverage area are seldom attended by a SALOHA system in high traffic conditions, because the probability of having another user closer to the BS is very high. So in our simulation a mobile in the limit of the coverage area will be always in retransmission mode which increases quickly the system delay. As the Bell Shape distribution reduces the probability of this type of mobiles, the throughput and the delay for this distribution are slightly better.

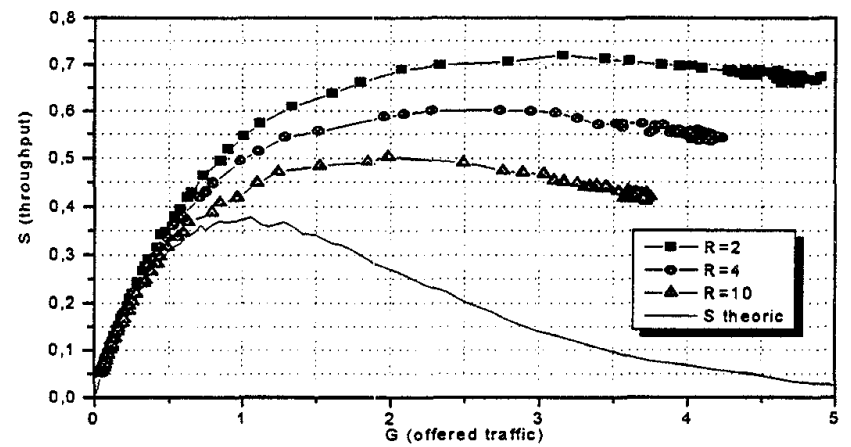

Figure 2. Throughput versus Offered Load for a Bell-Shaped Spatial Distribution for different capture ratios

In Figure 3 it can be seen that the throughput obtained with the unimodal distribution is lower than with the others as was expected. The differences start at an offered traffic around 1 packet per slot and increase considerable as traffic increases. But even in the case of having all the mobiles at the same distance from BS there is a. significant throughput (higher than in the case of no channel) due to channel capture effects.

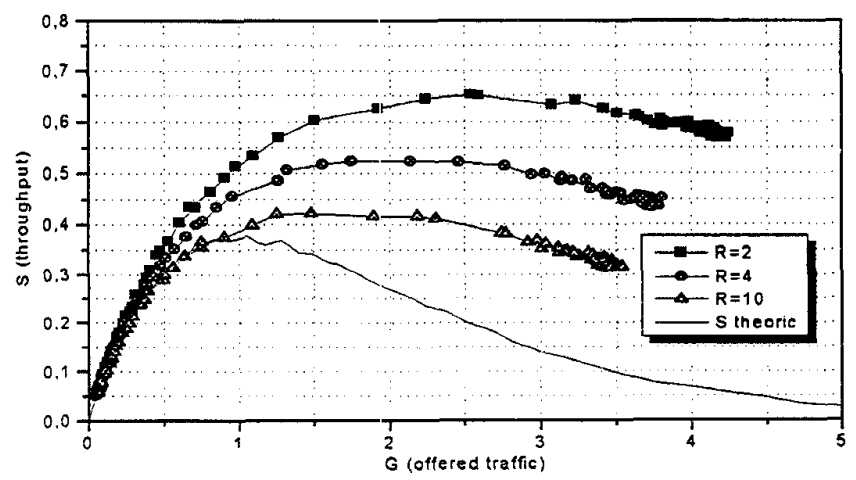

Figure 3. Throughput versus Offered Load for a Unimodal Spatial Distribution for different capture ratios .
In general, as it can be seen at the figures we can conclude that similar results have been obtained for the two spatial distributions with physical sense: Uniform and Bell Shape. So we can conclude that, as there are not significant differences between distributions, and being the uniform the easiest to implement, there is no necessity of trying another distributions to simulate the S-ALOHA system under real conditions.

According of such Figs. we can conclude that the difference between the throughput obtained by $\mathrm{R}=2$ and $R=10$ are very significant and we confirm the idea that higher values of $R$ produce worse stability conditions. The improvement in the throughput is mainly due to the real channel effects, because collision and therefore retransmission are only required when two or more packets with similar power levels are simultaneously transmitted.

Next three figures show the Delay versus Throughput behaviour for the three spatial distributions mentioned before and for different values of the capture ratio. The results are compared again with the theoretical S-ALOHA (without channel an with retransmission probability equal to new packet generation probability).

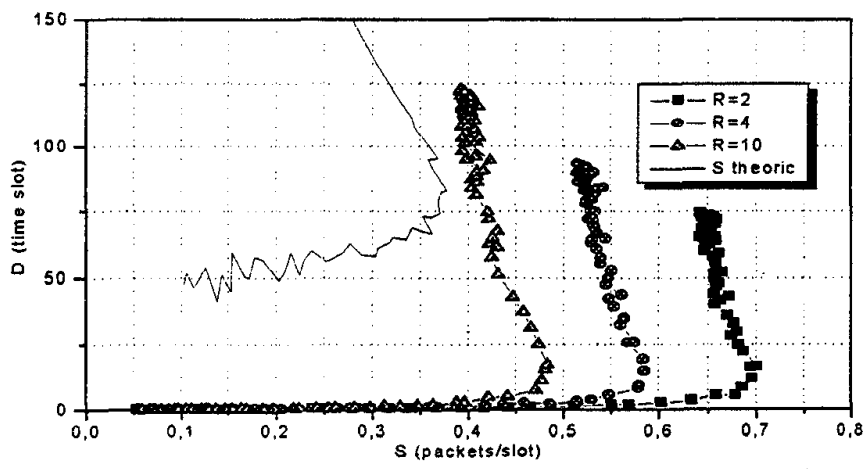

Figure 4. Delay (D) versus Throughput (S) for a Uniform Spatial Distribution for different capture ratio $(R)$

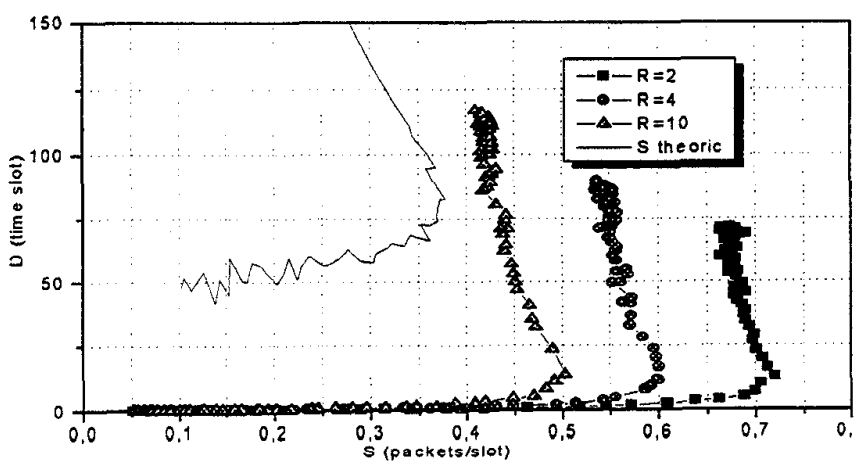

Figure 5. Delay (D) versus Throughput (S) for a Bell-Shaped Spatial Distribution for different capture ratio (R). 


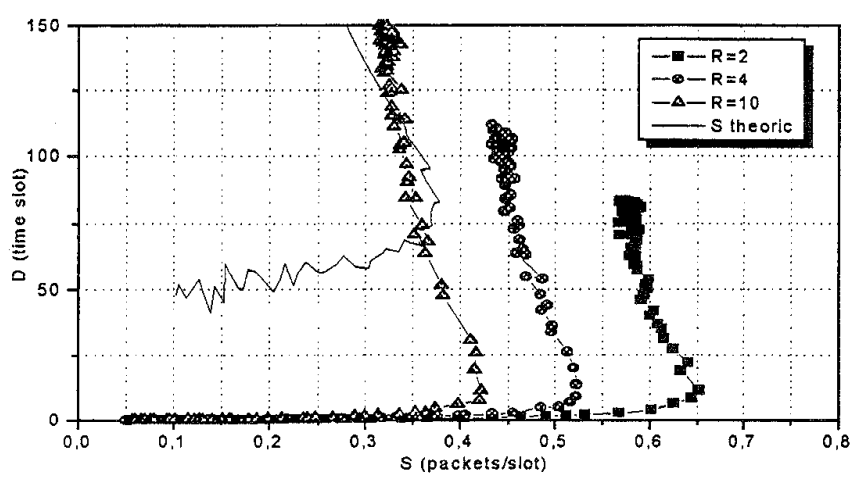

Figure 6. Delay (D) versus Throughput (S) for a Unimodal Spatial Distribution for different capture ratio (R).

The delay in number of timeslots (D) is very sensitive to the capture ratio values $(R)$ used in the simulation. The delays are not very significant until the maximum throughput is achieved. Then as the traffic continues growing the delay starts to increase very quickly. Comparing with the ideal S_ALOHA there is a significant reduction in the delay due both to the capture effect and to the retransmission technique used in the simulation.

\section{CONCLUSIONS}

Slotted Aloha with different spatial distributions under real channel environment have been studied, to determine how the Capture Effect can improve global system behaviour. The results of the analysis show that the Capture Ratio has a significance influence both in the Throughput and in the Delay independently of the Spatial Distribution considered. Higher throughput with a minimum delay and with stability under high traffic conditions can be achieved when compared to theoretical S-Aloha.

From the three spatial distributions under study the Bell Shape distribution represents the best choose because it offers slightly higher throughput and lower delays when compared to the other two. Moreover the Bell Shape distribution model is closer to the real mobile distribution in a cell because in the limits of distance of the cell many mobiles would be served by other BS (cell overlapping) and then the mobile density should be considered lower. Even for the worst spatial distribution, this is the unimodal one, the throughput and delay characteristics are significantly better than in the theoretical S-Aloha.

\section{REFERENCES}

[1] B. Hajek and T.van Loon, "Decentralized Dynamic Control of a Multiple access Broadcast Channel", IEEE Transactions on Automatic Control, Vol. AC-27, No. 3, pp.559-569, June 1982.
[2] D.Bertsekas and R.Gallager, "Data Networks", Englewood Cliffs, Prentice -Hall, 1987.

[3] Jean-Paul-Linnartz, "Narrow Band Land-Mobile Radio Networks", Artech House ed., 1993.

[4] J.C.Ambak and W.van Blitterswijk, "Capacity of Slotted ALOHA in Rayleigh-Fading Channels", IEEE Journal on Selected Areas in Communications, vol. SAC-5, No.2, pp.261- 269 February 1987

[5] Michele Zorzi, "Capture Probabilities in Random-Access Mobile Communications in the Presence of Rician Fading", IEEE Transactions on Vehicular Technology, vol.46, No.1, pp. 96-101, February 1997.

[6] C.van Der Plas and J.-P. M.G. Linnartz "The Near/Far Effect in ALOHA Radio Communications", IEEE Transactions on Vehicular Technology, vol. -39 , No.4, pp.359-366, November 1990.

[7] M.Zorzi and R.R.Rao, "Capture and Retransmission Control in Mobile Radio“, IEEE Journal on Selected Areas in Communications, vol. 12, No.8, pp. 1289-1298, October 1994.

[8] D.J. Jeong and W.S.Jeon, "Performance of an Exponential Backoff Scheme for Slotted-ALOHA. Protocol in Local Wireless Environment", IEEE Transactions on Vehicular Technology, Vol. 44, No. 3, pp.470-479, August 1995.

[9] D.Covarrubias, S.Ruiz, J.Huguet and J.Olmos, "Performance Analysis of SLOTTED-ALOHA Considering Different Retransmission Algorithms for Wireless Networks Under Real Channel Models", to be published at the proceedings of the International Conference Telecommunications 1998 ICT'98,june 1998 .

[10] Ch.T.Lau and C.Leung, "Capture Models for Mobile Packet Radio Networks", IEEE Transactions on Vehicular Technology, Vol. 40, No. 5, pp. 917-925, May 1992.

[11] V.Wong and C.Leung," "Capture Probability in a Mobile Packet Radio System", IEEE Transactions on Vehicular Technology, Vol. 40, No. 10, pp.1577- 1580, October 1992.

[12] S.A.Musa, and W.Wasylkiwskyj "Co-Channel Interference of Spread Spectrum Systems in a Multiple User Environment", IEEE Transactions on Communications, vol. COM-26, No.10, ,pp.1405-1413 October 1978.

[13] A.U.Sheikh, Y.D. Yao, and X. Wu, "The ALOHA Systems in Shadowed Mobile Radio Channels with Slow or Fast Fading", IEEE Transactions on Vehicular Technology, vol. 19, No. 4, pp.289-298, November 1990. 